\title{
Determining the endurance limit of AISI 4340 steels in terms of different statistical approaches
}

\author{
Salim Çalışkan, Rıza Gürbüz \\ Department of Metallurgical and Materials Engineering, Middle East Technical University, 06800 Ankara, Turkey \\ caliskan.salim@metu.edu.tr, bttps://orcid.org/0000-0002-9276-8492 \\ rgurbuz@metu.edu.tr, bttps://orcid.org/0000-0002-8369-4639
}

\begin{abstract}
In engineering applications, fatigue phenomenon is a key issue and needs to be analyzed in the beginning of design phase in case of any component exposed to alternating loading on operation otherwise catastrophic fatigue failure may cause. Component can be designed with safelife, fail-safe, and damage tolerant approach based on whether redundant load path and damage sensitive. Before starting analyzing the structure, material allowable data needs to be presented in a reliable way to predict fatigue life of components. SN curves with presented confidence levels are the robust approach to make a prediction on safe life of a structure in terms of fatigue. In this point, there are so many approaches to determine fatigue limit of materials and issue shall be handled by statistical manner. In literature, different staircase and curve fitting methods were presented to estimate endurance limit of materials and some reliability manuscript published. In this paper, fatigue limit of AISI 4340 steel will be investigated through most convinced staircase and curve fitting approaches and their reliability will be queried.
\end{abstract}

KEYwORDS. Steels; Fatigue; Staircase; Curve Fitting; Reliability

\begin{abstract}
OPEN ACCESS
Citation: Çalışkan, S., Gürbüz, R., Determining the endurance limit of AISI 4340 steels in terms of different statistical approaches, Frattura ed Integrità Strutturale, 58 (2021) 344-364.

Received: 14.07.2021

Accepted: 13.09 .2021

Published: 01.10 .2021

Copyright: (C) 2021 This is an open access article under the terms of the CC-BY 4.0, which permits unrestricted use, distribution, and reproduction in any medium, provided the original author and source are credited.
\end{abstract}

\section{INTRODUCTION}

$\mathrm{S}$ $\mathrm{N}$ curves have been firstly used by German engineer, Wöhler and stress-life was formulated in linear equation; hereby, logarithm of life is correlated with oscillating stress [1]. Then, Basquin proposed new model by using each side natural logarithm of stress and life [2]. However, addition of initial boundary data point (ultimate stress) to SN curve resulted in poor estimation. So, non-linear model was presented by Stromeyer by smoothing previous models [3]. To enhance best fit of SN curve, researchers continued to propose new models basically same approach stated above by adding parameters or changing complexity of the relation. Some of them are accepted as common standard in constructing of SN curves. In the literature, many approaches have been presented best fit of data with large number of specimens however inconsistency results in case of small specimen data sets [4]. Even though new technologies enable performing fatigue tests with ultra- 
high-test frequency on resonant test systems these days, SN curve models need to be compatible with small data sets due to time limitation for UHCF [5].

Fatigue failure caused by surface feature is attributed to crack initiation; on the other hand, crack propagation is because of bulk characteristic of material. Therefore, crack is easily formed at high stress values and resulted as less scatter; however, large scatter is observed around fatigue limit because of local circumstances in the reverse condition [6]. Although coupon level testing provides information about scatter, it can be difficult to predict in service conditions. SN curves are affected by grain size and direction of grains, composition of material, surface finish after machining and heat treatment [7]. Therefore, scatter caused by variation in material and production-based reasons cannot be overestimated for products in service and needs to be verified by component level testing. It is commonly used method to perform tests at least twice covering operation life in service in order to observe critical locations in terms of crack susceptibility. In literature, scatter effect is covered by confidence levels by statistical approaches [8].

Unless specified SN curves in the literature presented as mean curve with 50\% quantile. Designers use $95 \%$ confidence level to be sure safe fatigue limit and 95\% meets their requirements [9]. Design curves are commonly obtained by curve fitting methods based on log-normal or Weibull distribution. SN curve fitting can be evaluated simply through two parameters because of not dealing with complexity and it offers well suited and results are adequately accurate [10]. Fatigue data of metallic materials exhibit log-normal or Weibull distribution based on analytic equations with certain inconsistency; therefore, all approaches need to verify by fatigue testing to get reliable data [11].

Even though fatigue strength exhibits normal distribution extensively, coefficient of variance in fatigue limit reflects lognormal distribution and scatter is greater than initiation dominant life region compared to propagation one that is under high stress loading. Previous one is well fitted with Weibull distribution and latter is exhibited by log-normal distribution. Standard deviation changes with applied stress; accordingly, decreasing stress or strain results in increasing standard deviation. Maximum likelihood method covers run-out data points by linear regression to estimate observed data. Least square estimation is a method that minimizes the residuals (sum of squared deviation) of experimental data. For smooth specimen run-out criteria can be chosen as $2 \mathrm{E}+06$ cycles because of not existing stress concentration [12]. Barbosa et al. studied different estimated fatigue models to exhibit good adjustment between observed and fitted data. Difference of this models is to provide best fit from ultra-low cycles to high cycles; however, medium and high cycle region is the focus for design issue for a structure. Schijve et al. investigated fatigue limits statistical point of view and resulted in Weibull is better suited than log-normal distribution. Another approach is proposed by Stüssi and non-linear equation is formulated with stress and life relation taking ultimate and infinite endurance stress into formulation. By linear regression, model parameters can be estimated however combined Stüssi and Weibull distribution approach which is proposed by Caiza et al. results in good fit in all regions of SN curve due to better estimation of model parameter [13]. Burhan et al. also investigated different curve fitting methods for fatigue data characterization in terms of competence of SN curve, estimation of fitting parameter consistency, adjustment to other stress ratios, presented as fatigue damage estimation and curve shape ability covering ultimate stress point. Kim et al. found that micro-crack density scales up logarithmically with fatigue cycles increases rather than micro-crack length [14]. Differently, Lipski et al. studied constructing SN curve based on thermography analysis and provides an advantage to stop test before catastrophic failure based on temperature change [15]. ASTM E739-91 proposes simple linear relation between stress and life in log scale. Model uses log-normal distribution and it can be solved by linear regression based on Maximum likelihood approach. It also gives information about how many specimens are needed to get reliable data. Accordingly, recommended data set shall be between 12-24 specimens. As a guideline, confidence level shall be $5 \%$ and $95 \%$ to estimate confidence band that covers observed experimental data. Because of limitation of model, very low cycle regions are out of consideration and therefore ASTM E739-91 model is suitable for medium and high cycle fatigue regions.

According to Dixon, the idea behind of staircase is based on concentrating of data points around mean value. Sample size needs to be large enough accordingly Dixon suggested at least 40 specimens are required for accurate data evaluation. Increment range shall be between $0.5 \sigma$ and $2 \sigma$ for good estimation if standard deviation is known; otherwise, small interval results in spending time unless starting point close to mean value [16]. This approach is modified by Dixon and proposed that increment step can be estimated as $\sigma$ and maximum $50 \%$ error of standard deviation can be tolerated. Therefore, increment step may be $2 / 3 \sigma$ and $3 / 2 \sigma$ for likely guess since small interval produces smaller covariance in case of given mean value previously; however, large step size results in decreased number of trials around mean value [17]. Even though it is recommended to make analysis through 15 specimens by staircase method, test set is generally managed by 6 specimens many discussions are available to estimate fatigue limit of material with less number by preserving reliability of data [18]. Fatigue limit of material generally follows normal distribution. In engineering applications, it also exhibits log-normal distribution. Primary condition of staircase method is to estimate by normal distribution; however, safety factor needs to be evaluated through log-normal distribution to prevent oversizing. 
According to Zheng's study, intermediate and long-life regions of SN curve follow normal and log-normal distribution respectively. Rabb studied whether starting point has effect on staircase method or not and resulted as little influence. The most popular approach is starting with estimated fatigue limit to increase accuracy of analysis.

In case logarithmic stress values are equally spaced, the log-normal and Weibull distribution can be used as an analyzing of staircase method. To estimate mean value and confidence level of fatigue data, choosing increment level between $2 / 3 \sigma$ and $3 / 2 \sigma$ provides well-established analysis [19]. Basically, it can be assumed that there exists threshold above fatigue limit and facilitates crack initiation under applying higher stress than endurance limit [20].

Reduction of fatigue limit requires knowledge of mean strength and standard error. Although fatigue strength is insensitive to increment size, standard deviation is affected markedly. The value of coefficient of variation is a key parameter for fatigue limit analysis since almost similar results rise for low values of $\mathrm{CoV}$ but not satisfactory results for high values therefore Dixon-Mood is the best option in this case [21]. Lin at al studied that method used to determine endurance limit can be chosen based on covariance of data; in case of small covariance ray projected method (extrapolating through low cycles data) gives better result but Dixon-Mood method (based on Maximum Likelihood Estimate) is advised for large covariance. However, projection method is not reliable below $10^{4}$ cycles therefore stress level is critical for accelerated analyses. Even though most popular approach is Maximum Likelihood Estimate for staircase method, Wallin justify this approach because scatter exhibits Weibull distribution not normal distribution which is underlying assumption for MLE.

Monte Carlo method is proposed by iterating of data points in terms of arithmetical operation [22]. Similarly, Pollak et al. used bootstrapping method to decrease standard error for small sample data sets as an alternative to Dixon-Mood method since latter method gives accurate results in case of large specimens (at least 40 specimens) however underestimated results with small data sets. Bootstrap is an alternative method by counting standard deviation and confidence level around the mean value. The advantage is no need to use distribution hypothesis like classical methods nevertheless it presents same results and data points develop with replacing data points through replication [23]. As different approach, fatigue strength of material is governed by inhomogeneous distribution of different types of defects. It has proved that non-propagating cracks ahead of micro-notches exist below the stress level of endurance limit. Based on Murakami and Endo assumption, analysis for fatigue limit gave similar results for annealed condition steel; however, it will be different for hardened steel because of non-metallic inclusions effect. Beretta et al. studied fatigue properties in terms of statistical manner and minimum number of defects inside microstructure and resulted as maximum likelihood is the best estimator because of having less standard deviation of excessive defects [24].

\section{EXPERIMENTAL PROCEDURE}

\footnotetext{
A ISI 4340 steel with a composition (in wt $\%$ ) of $.38 \% \mathrm{C}, .65 \% \mathrm{Mn}, .7 \% \mathrm{Cr}, 1.65 \% \mathrm{Ni}, .2 \% \mathrm{Mo}, .025 \% \mathrm{P}$ and $\mathrm{S}$, bal. $\mathrm{Fe}$, was chosen as a research material because it exhibits a constant endurance limit after a certain fatigue life. Additionally, it shows high fatigue strength and toughness therefore it is considered as a good candidate for aircraft material. A set of smooth round fatigue specimens with $22 \mathrm{~mm}$ gage diameter and $130 \mathrm{~mm}$ length was manufactured from AISI 4340 steel with optimum machining parameters. Before specimen preparation, material was heat treated per AMS 2759-1D for optimum condition for machining. Firstly, material was normalized at $899^{\circ} \mathrm{C}$ for 90 minutes then austenitized at $816^{\circ} \mathrm{C}$ for 60 minutes and finally tempered at $593^{\circ} \mathrm{C}$ for 3 hours. Normalizing removed internal stress and provided microstructural homogeneity. Austenitizing and tempered provided desired microstructure in terms of strength and hardness. After quenching, martensite phase forms because of not allowing enough time for diffusion of carbon atoms because of rapid cooling and resulting BCT crystal structure. Later, tempering results in bainite (intermediate phase) and ferrite phases in the microstructure by allowing transformation of martensite to bainite phase. As a result, crystal structure is composed of BCC after this transformation. The microstructure of material is given on Fig. 1. Mechanical properties of material results in $1080 \mathrm{MPa}$ ultimate strength and $29 \mathrm{HRC}$ hardness after heat treatment process.

A total of 14 specimens were used to determine endurance limit of material. All of them were out from Rolling DirectionR90 orientation. Crack initiation was observed in R90-Transverse plane which is perpendicular to applied stress along Rolling Direction.

In this research, $\mathrm{SN}$ curves were constructed by using different methods providing data points for finite and infinite life regions. Entirely 14 specimens were sequentially tested, 8 of them were assigned for establishing endurance limit of material with applying less stress range and 6 of them were used to focus on finite fatigue region. Fatigue tests were performed under constant amplitude cyclic loading in laboratory conditions $\left(23 \pm 2^{\circ} \mathrm{C}\right.$ and $\left.50 \pm 5 \mathrm{RH}\right)$ per ASTM E466-96. Round fatigue specimens were alternately axial loaded by applying sinusoidal wave form with stress ratio $\mathrm{R}$ of 0.1 (tension-tension) under constant $150 \mathrm{~Hz}$ test frequency on RUMUL resonant test system.
} 


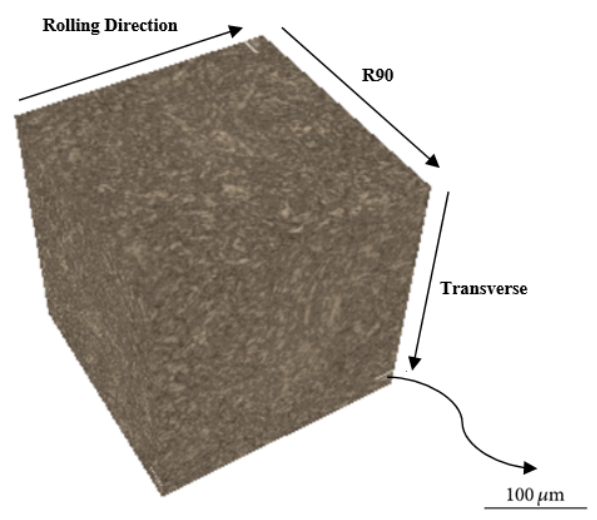

Figure 1: Micrograph of AISI 4340 steel

\section{Staircase Analysis}

Staircase or up-and-down method is commonly used method for determining fatigue limit of material at a specified life value. Accordingly, run-out criterion is firstly stated (say $10^{7} \mathrm{cycles}$ ) and first specimen is roughly tested around mean value then if the specimen failed at this stress level. Next specimen will be tested at less stress level with defined stress increment otherwise, stress level increased in case of run-out case. Hence, applied stress for next specimen depends on foregoing test results. Stress increment is determined based on standard deviation and rest of the specimens are tested in this manner.

Dixon-Mood Method

Dixon-Mood method derived from Maximum Likelihood theory is a common approach to determine endurance limit of materials. Tests are carried out by increasing or decreasing stress level with defined increment based on standard deviation and generated the sequence also called up-down method. The purpose is to focus around median value to determine accurate fatigue strength with defined confidence levels. Sample mean and standard deviation can be estimated by given equations:

$$
\begin{aligned}
& S=S_{0}+d \cdot\left(\frac{A}{F}-\frac{1}{2}\right) \quad \text { when less periodic event becomes failures } \\
& S=S_{0}+d \cdot\left(\frac{A}{F}+\frac{1}{2}\right) \quad \text { when less periodic event becomes run-outs } \\
& s=1.62 \cdot d \cdot\left(\frac{F \cdot B-A^{2}}{F^{2}}+0.029\right) \text { n case of } \frac{B \cdot F-A^{2}}{F^{2}}>0.3 \\
& s=0.53 \cdot d . \text { in case of } \frac{B \cdot F-A^{2}}{F^{2}}<0.3
\end{aligned}
$$

where $S_{0}$ is the lowest stress level of dataset, $d$ is the stress increment, $F=\sum \mathrm{f}_{\mathrm{i}}, A=\sum \mathrm{i} \cdot \mathrm{f}_{\mathrm{i}}, B=\sum \mathrm{i}^{2} \cdot \mathrm{f}_{\mathrm{i}}, \mathrm{i}$ is the numbering of stress level and $f_{i}$ is the number of samples corresponding to the stress level.

If estimated stress increment is extremely higher than standard deviation; then sample mean will be higher than reality and standard deviation will be less. In case of reverse condition, higher standard error and lower sample mean will be obtained. It was expected to choose stress increment around $2 / 3 \sigma$ and $3 / 2 \sigma$ before starting test.

Sample mean with 50\% confidence level and standard deviation for Dixon-Mood method can be computed as $585 \mathrm{MPa}$ and 23.21 MPa based on equations given above and variables can be seen on Fig. 2. Mean value needs to be defined with confidence interval since every set of test specimen will give different mean values because of scatter and confidence interval for sample mean will provide to present conservative data for endurance limit. It can be used that $95 \%$ confidence interval for sample mean to stay safe side meaning that $95 \%$ of test results will be upwards of mean value. Confidence interval with unknown coefficient of variance is distributed per t-distribution meaning that defined confidence interval $((1-\alpha) \cdot 100 \%)$ is symmetric around empirical sample mean and underside boundary can be expressed as given equation below:

$$
S_{X \%}=S-t_{\alpha, n-1} \cdot \frac{s}{\sqrt{ } n}
$$




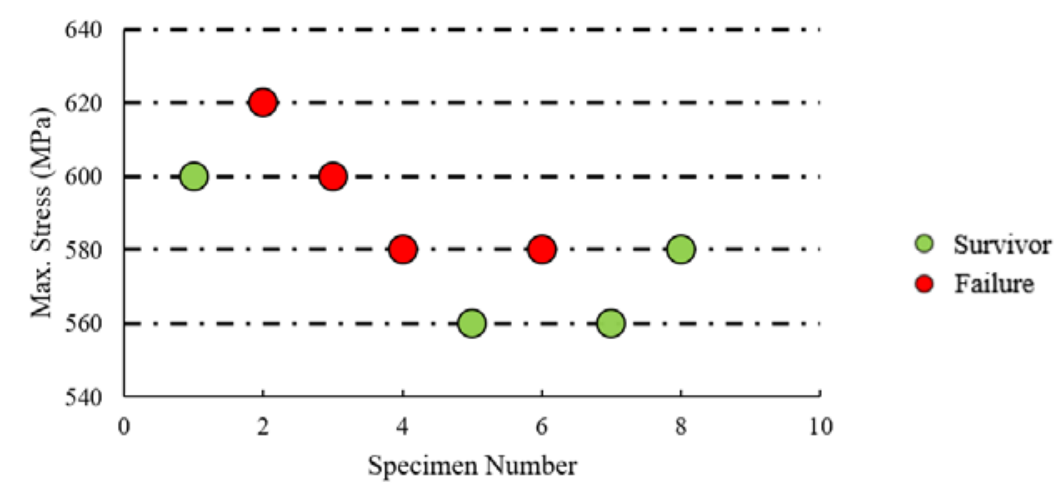

\begin{tabular}{|c|c|c|c|}
\hline $\mathrm{i}$ & $\mathrm{f}_{\mathrm{i}}$ & $\mathrm{i}^{*} \mathrm{f}_{\mathbf{1}}$ & $\mathrm{i}^{2 *} \mathrm{f}_{\mathrm{i}}$ \\
\hline 2 & 1 & 2 & 4 \\
\hline 1 & 1 & 1 & 1 \\
\hline 0 & 2 & 0 & 0 \\
\hline$\Sigma$ & 4 & 3 & 5 \\
\hline & $\mathbf{F}$ & $\mathbf{A}$ & $\mathbf{B}$ \\
\hline
\end{tabular}

Figure 2: Staircase Sequence per Dixon-Mood Method-

In this way, limits of probability of failure are taken into consideration with reduced endurance limit. Accordingly, mean value with $95 \%$ confidence interval can be calculated as $563.8 \mathrm{MPa}$.

Similarly, confidence interval for standard deviation needs to be calculated to validate standard deviation below the upper limit of empirical standard deviation coming from fatigue test data with defined confidence level. It obeys chi-square right tailed probability of distribution and it is calculated by given equation:

$$
s_{X \%}=\sqrt{\frac{n-1}{\chi_{\alpha, n-1}^{2}}} \cdot s
$$

Eventually, standard deviation with 95\% confidence interval is found to be $56.92 \mathrm{MPa}$ as shown in Fig. 3. This value will be used to determine endurance limit of material as the limits for probability of failure taking place.

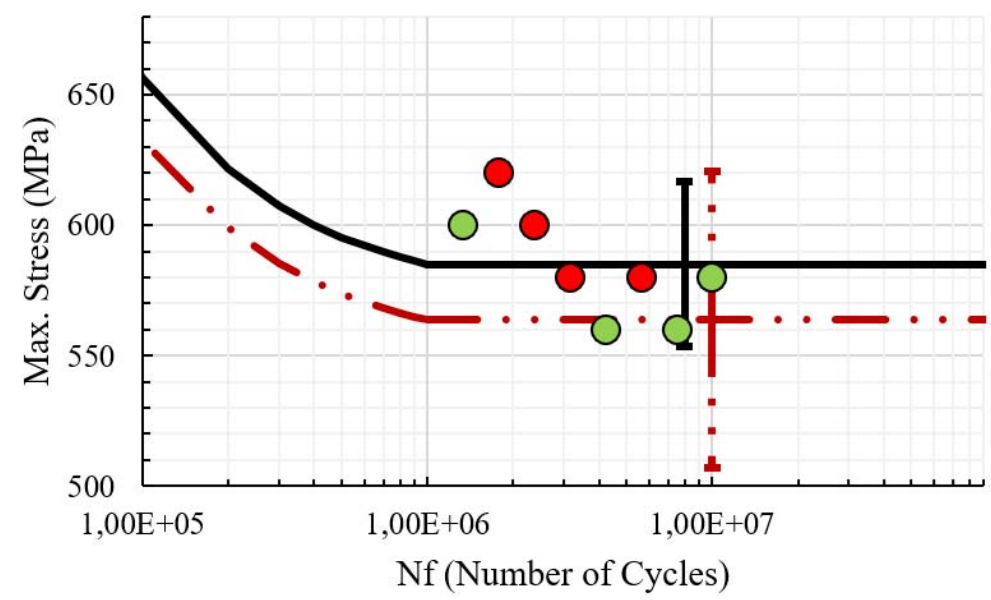

Figure 3: Mean value for 50\% probability of failure and 95\% confidence level with given standard deviations.

\section{$\underline{\text { IABG Method }}$}

Another approach for staircase method is IABG method proposed by Hück [26]. Accordingly, it is like Dixon-Mood method in terms of evaluation of data. IABG method starts with second test specimens' data and first one is discarded and not evaluated rather fictitious data is added at the end of data set as shown in Fig. 4. Additionally, evaluation cover all failed and survived specimens on specified stress range and based on logarithmic values of data. Same data above will be evaluated in same manner with given equations presented by IABG method [27].

$$
\log (S)=\log \left(S_{0}\right)+\log (d) \cdot \frac{A}{F}
$$




$$
\begin{aligned}
& k=\left\{\begin{array}{l}
\frac{F^{*} B-A^{2}}{F^{2}} \\
e s e-5
\end{array} \cdot \frac{F \cdot B-A^{2}}{F^{2}} \geq 0.5 ; a=4.579494 \cdot F^{-0.889521} ; b=7.235548 \cdot F^{-0.405229}\right. \\
& \log (\log (s))=\log (d) \cdot 10^{a} \cdot k^{b}
\end{aligned}
$$

Hereby, $S$ and $s$ are the mean value and standard deviation of data set respectively, $d$ is stress increment on logarithmic base and $k, a$ and $b$ are the auxiliary variables.

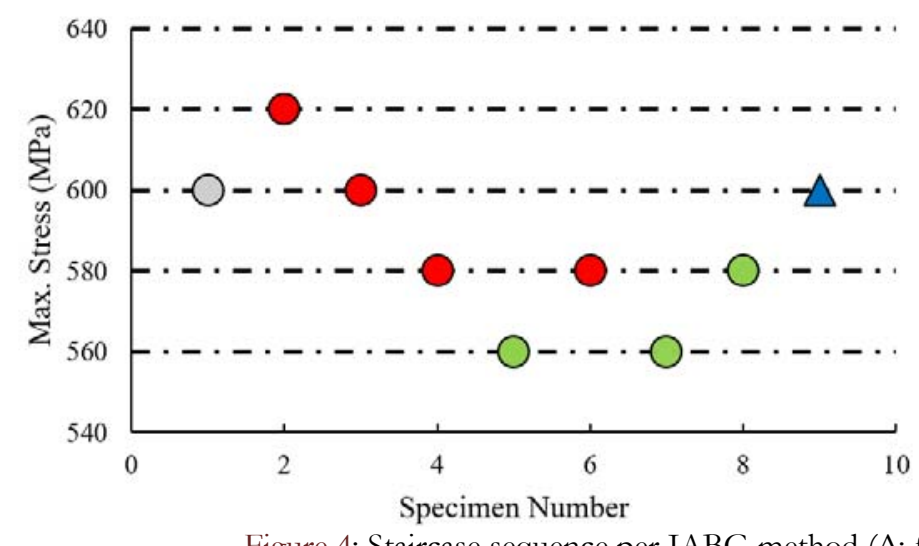

\begin{tabular}{|c|c|c|c|}
\hline $\mathrm{i}$ & $\mathrm{f}_{i}$ & $\mathrm{i}^{*} \mathrm{f}_{i}$ & $\mathrm{i}^{2 * \mathrm{f}_{i}}$ \\
\hline 3 & 1 & 3 & 9 \\
\hline 2 & 2 & 4 & 8 \\
\hline 1 & 3 & 3 & 3 \\
\hline 0 & 2 & 0 & 0 \\
\hline$\Sigma$ & 8 & 10 & 20 \\
\hline & $\mathbf{F}$ & $\mathbf{A}$ & $\mathbf{B}$ \\
\hline
\end{tabular}

Figure 4: Staircase sequence per IABG method ( $\Delta$ : fictitious data).

Mean value with $50 \%$ confidence level and standard deviation for IABG method can be computed as $585.11 \mathrm{MPa}$ and 14.54 $\mathrm{MPa}$, respectively based on equations specified above and logarithmically shown in Fig. 5.

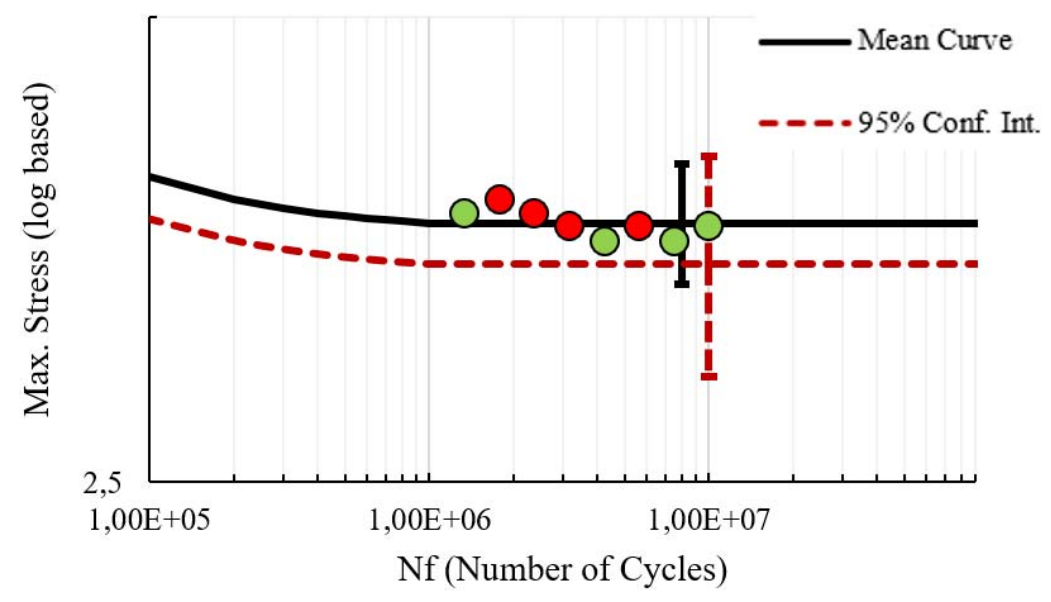

Figure 5: Mean value and standard deviation with given 95\% confidence level based on IABG method.

\section{Bootstrapping Method}

Another approach is bootstrapping method introduced by Efron [28] which is based on data simulation providing many unreal data in other words re-sampling from original data. Standard deviation of random distribution of a population is perpetually indeterminate; therefore, most popular approach is to use estimated standard error and correlating standard deviation. Since bootstrapping covers all present information about the distribution, it could be a good choice to estimate standard error of data set. Samples are normally distributed as shown in Fig. 6. The idea behind of approach is to decrease scatter by allowing less biased standard error. 


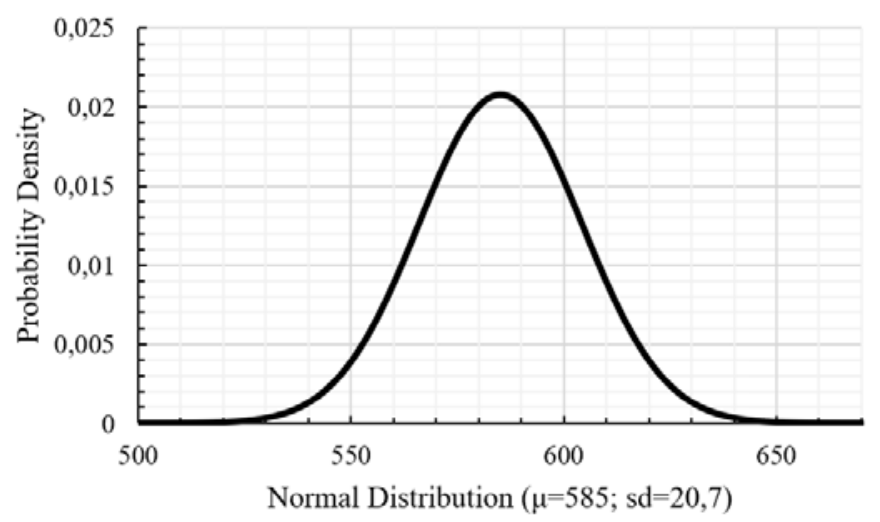

Figure 6: Normal distribution of staircase data based on Bootstrapping method.

Standard error estimation of re-sample distribution for bootstrapping method is originated by Monte-Carlo approach. Approximating population with larger sample size is advanced at least 200 times re-sampling from original data [28]. During analysis, 200 times original data was re-sampled and only 10 of them can be seen on Tab. 1.

\begin{tabular}{cccccccccc}
\hline $\mathrm{b}$ & $\mathrm{X}(1)$ & $\mathrm{X}(2)$ & $\mathrm{X}(3)$ & $\mathrm{X}(4)$ & $\mathrm{X}(5)$ & $\mathrm{X}(6)$ & $\mathrm{X}(7)$ & $\mathrm{X}(8)$ & $\mathrm{X}^{*}$ \\
1 & 580 & 580 & 600 & 580 & 580 & 580 & 600 & 600 & 587.5 \\
2 & 580 & 620 & 600 & 620 & 600 & 560 & 600 & 580 & 595 \\
3 & 580 & 580 & 560 & 620 & 580 & 620 & 560 & 620 & 590 \\
4 & 560 & 560 & 580 & 580 & 560 & 560 & 580 & 560 & 567.5 \\
5 & 560 & 600 & 600 & 580 & 560 & 580 & 580 & 560 & 577.5 \\
6 & 560 & 560 & 600 & 560 & 560 & 560 & 560 & 560 & 565 \\
7 & 580 & 580 & 580 & 600 & 560 & 560 & 580 & 580 & 577.5 \\
8 & 600 & 580 & 620 & 580 & 560 & 620 & 600 & 600 & 595 \\
9 & 620 & 560 & 600 & 600 & 600 & 560 & 600 & 600 & 592.5 \\
10 & 580 & 600 & 580 & 620 & 580 & 620 & 560 & 560 & 587.5 \\
\hline
\end{tabular}

Table 1: Bootstrapping Algorithm.

Mean value and estimated standard deviation of bootstrap replications can be seen from following equations:

$$
\begin{aligned}
& \bar{X}=\frac{1}{B} \sum_{i=1}^{B} X_{i}^{*} \\
& s=\left(\frac{1}{B-1} \sum_{i=1}^{B}\left(X_{i}^{*}-\bar{X}\right)^{2}\right)^{\frac{1}{2}}
\end{aligned}
$$

Similarly, standard deviation of Monte Carlo approximation based on bootstrapping replications can be calculated by following equation:

$$
s=\left(\frac{1}{n^{2}}\right) \sqrt{n}
$$

Standard deviation of 200 means is the bootstrapped standard error of the mean. Mean value can be calculated as 585.11 and estimated standard deviation is 7.09 after bootstrapping analysis in Fig. 7. Standard deviation based on Monte Carlo approximation is 9.19 which is close to bootstrapping method. By using bootstrapping re-sampling, confidence intervals 
could be established for given population. Bootstrap t-interval and standard confidence interval $(1-\alpha)$ can be found with given equations, respectively:

$$
\begin{aligned}
& t_{\alpha, n-1}=\frac{\left(\overline{\mathrm{X}}-X_{\text {lower }}\right)}{s} . \\
& X_{\text {lower }}=\overline{\mathrm{X}}-z_{\alpha / 2} * s
\end{aligned}
$$

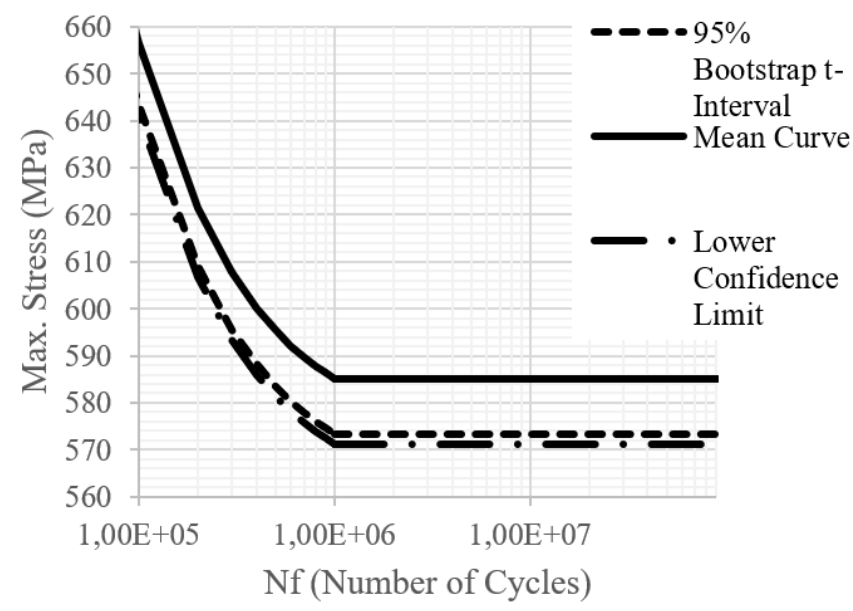

Figure 7: Mean curve (solid line) along with 95\% bootstrap t-interval (dash line) and standard (long dash dot) lower confidence limit

\section{Weibull Method}

Function of random variable with unknown parameter can be associated with fatigue data. Weibull distribution facilitates estimating low probability of failures compared to normal distribution and fatigue limit can also be obtained by 2-parameter Weibull distribution (location parameter not used). Most popular optimizing technique to estimate scale $(\alpha)$ and shape ( $\beta$, Weibull slope) parameters is maximum likelihood estimator by correlating variables with most likely observed data. All of data (failed and runout) can be evaluated during analysis and not necessarily to specify constant stress increment for MLE method. Therefore, the advance of MLE is treating censored data correctly with any distribution.

Maximized likelihood function for staircase tests is given in Eqn. 15.

$$
L=\prod_{i=1}^{n} F\left(S_{\alpha}^{i},\{p\}\right) \cdot \prod_{j=1}^{m}\left[1-F\left(S_{\alpha}^{j},\{p\}\right)\right]
$$

where $n$ and $m$ are failed and run-out specimens respectively, $\{p\}$ is the parameters describing distribution and $F$ is the cumulative density function.

By optimizing $L$, scale and shape parameters can be estimated using Newton Raphson method which is an iterative technique for Weibull distribution function. Accordingly, $\beta$ and $a$ can be estimated by given iteration method as shown in Fig. 8.

$$
\beta_{k+1}=\beta_{k}-\frac{\frac{1}{\beta}+\frac{\sum_{i=1}^{n} \ln x_{i}}{n}-\frac{\sum_{i=1}^{n} x_{i}^{\beta} \ln x_{i}}{\sum_{i=1}^{n} x_{i}^{\beta}}}{\left(-\frac{1}{\beta^{2}}+\frac{\left(\sum_{i=1}^{n} x_{i}^{\beta} \ln x_{i}\right)^{2}}{\left(\sum_{i=1}^{n} x_{i}^{\beta}\right)^{2}}-\frac{\sum_{i=1}^{n} x_{i}^{\beta}\left(\ln x_{i}\right)^{2}}{\sum_{i=1}^{n} x_{i}^{\beta}}\right)}
$$




$$
\begin{aligned}
& \alpha=\left(\frac{\sum_{i=1}^{n} x_{i}^{\beta}}{n}\right)^{1 / \beta} \\
& \mu=\alpha \Gamma\left(1+\frac{1}{\beta}\right) \\
& \sigma=\left(\alpha^{2} \Gamma\left(1+\frac{2}{\beta}\right)-\mu^{2}\right)^{1 / 2}
\end{aligned}
$$

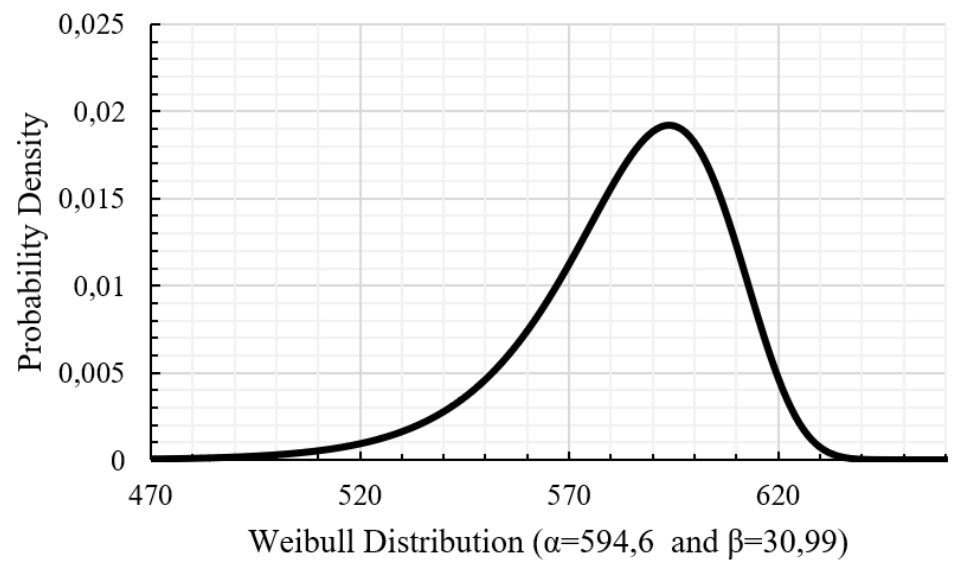

Figure 8: Weibull distribution of staircase data based on Newton Rapson technique.

Mean value and standard deviation can be estimated 584.13 and 23.64 based on Weibull distribution.

\section{ISME Method}

Another method used as a standard that is JSME (Japan Society of Mechanical Engineers) requiring 14 specimens for analysis and constructs whole of the SN curve. Eight of them are used in linear region and six specimens are enough to determine fatigue limit of data set. First stress point is taken as highest non-failed (run-out) point in data set. Rest of them is constructed up-and-down method based on previous test result by a certain stress increment. Stress increment for staircase sequence is estimated by assuming standard deviation equals to stress increment. Linear region is associated with following parameters:

$$
\begin{aligned}
& \log (N)=\alpha+\beta * S \\
& \beta=\frac{\sum_{i=1}^{8}\left(S_{i}-\bar{S}\right) *\left(\log \left(N_{i}\right)-\log (\bar{N})\right.}{\sum_{i=1}^{8}\left(S_{i}-\bar{S}\right)^{2}}, \log (\bar{N})=\frac{1}{8} \sum_{i=1}^{8} \log \left(N_{i}\right) \text { and } \bar{S}=\frac{1}{8} \sum_{i=1}^{8} S_{i}
\end{aligned}
$$

Mean and standard deviation can be estimated with given equations:

$$
\begin{aligned}
& S=\frac{1}{6} \sum_{j=2}^{7} S_{j} \\
& s=\frac{1}{|\beta|} \sqrt{\frac{1}{6} \cdot \sum_{i=1}^{8}\left(\operatorname { l o g } \left(N_{i}-\left(\alpha+\beta \cdot \log \left(S_{i}\right)\right)^{2}\right.\right.}
\end{aligned}
$$

After calculation, mean and standard deviation were calculated as 583.3 and 26.01 respectively. 
Curve Fitting Analysis

In engineering applications, SN curves are constructed by linear regression of stress versus life data with given standard deviation. This procedure is also known as curve fitting of fatigue data by executing goodness-of-fit. Weibull and log-normal distribution are suitable for metallic materials data to determine life of material. Life prediction is carried out by modelling of SN curve and constructing design curves with estimated confidence band, then fatigue limit is estimated based on probabilistic fatigue life model.

Basquin Method

Firstly, Wöhler formulated SN curve with regression parameters to get linear relationship between stress and life. SN curve in finite region is represented by linear correlation between stress and life in logarithmic scale. Then, another and most common approach was proposed by Basquin and it interrelates stress as a function of life.

$$
\begin{aligned}
& \sigma_{a}=\sigma_{f} \cdot\left(2 N_{f}\right)^{b} \\
& \operatorname{CoV}=\sqrt{10^{b^{2} s^{2}-1}}
\end{aligned}
$$

These equations based on log-normal distribution, $\sigma_{a}, \sigma_{f}$, and $b$ are the applied stress, fatigue strength coefficient and fatigue strength exponent respectively. Coefficient of variance is calculated by correlation of fatigue strength coefficient with standard deviation $(s)$. Standard deviation is estimated by $\log (2 \mathrm{~N} f)$ over $\log \left(\sigma_{a}\right)$. By using least square method, SN curve with finite life can be estimated with $50 \%$ reliability. Accordingly, mean value for $\% 50$ probability of failure in $10^{7}$ cycles (assumed as run-out criterion) and standard deviation are calculated as $592.89 \mathrm{MPa}$ and 34.7 respectively as shown in Fig. 9 .

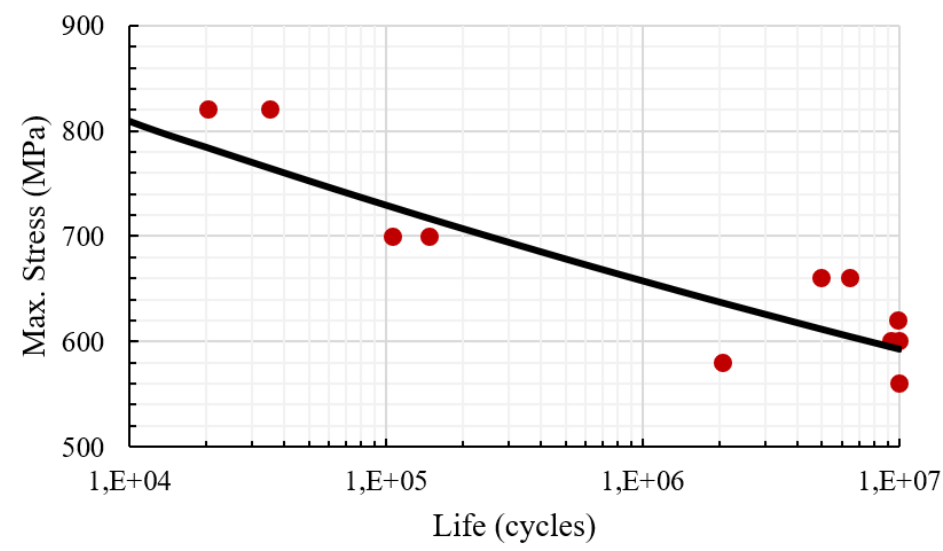

Figure 9: SN curve per Basquin method.

Stromeyer proposed a new equation for estimating stress by addition of $\sigma_{\infty}$ term even though endurance limit at defined $\sigma_{\infty}$ not exist.

$$
\sigma=\alpha \cdot\left(N_{f}\right)^{\beta}+\sigma_{\infty}
$$

where $\sigma$ is applied stress, $a$ and $\beta$ are fitting parameters, $N_{f}$ is the number of cycles and $\sigma_{\infty}$ is stress at infinity.

Based on this assumption, Stromeyer's model is not linear as in the case of Basquin. It practically smooths linear methods by adding new parameter that makes estimation more complicated. Similarly, Palmgren and Weibull later proposed new methods by addition of a dependent term and static stress respectively [3]. The idea behind these models is to provide smoother curve with given fitting parameters to estimate fatigue behavior of materials.

ASTM E739-91 Method

ASTM E739-91 defines a correlation between stress and life in linear region by sizing experimental data not extrapolating outside the data with given confidence intervals. The linear relation is established with failed specimens (run-out not included) and evaluated on logarithmic scale. It suggests that at least 12 specimens are required to get reliable data for allowable purposes [29]. 


$$
\begin{aligned}
& \log (N)=a+b * \log (\sigma) \\
& b=\frac{\sum_{i=1}^{n}\left(\log \left(\sigma_{i}\right)-\log (\bar{\sigma})\right)^{*}\left(\log \left(N_{i}\right)-\log (\bar{N})\right)}{\sum_{i=1}^{n}\left(\log \left(\sigma_{i}\right)-\log (\bar{\sigma})\right)^{2}} \\
& s=\frac{\sum_{i=1}^{n}\left(\log \left(N_{i}\right)-\log (\bar{N})\right)^{2}}{(n-2)}
\end{aligned}
$$

In given equations, life $(\mathrm{N})$ is the random variable, $\mathrm{a}$ and $\mathrm{b}$ are the fitting parameters, $\mathrm{n}$ is number of specimens, $\mathrm{s}$ is standard deviation and stress $(\sigma)$ is the independent variable. $\bar{\sigma}$ and $\bar{N}$ are mean values for stress and life respectively. Eventually, linear fit is estimated by regression.

The confidence intervals for entire SN curve are estimated for 90 and $95 \%$ probability of failure.

$$
a+b \cdot \log (\sigma) \pm \sqrt{2 \cdot F_{p}} \cdot s \cdot\left[\frac{1}{n}+\frac{(\log (\sigma)-\log (\bar{\sigma}))^{2}}{\sum_{i=1}^{n}\left(\log \left(\sigma_{i}\right)-\log (\bar{\sigma})\right)^{2}}\right]^{1 / 2}
$$

where Snedecor distribution value $\left(F_{p}\right)$ is tabulated per degree of freedoms in ASTM E739-91.

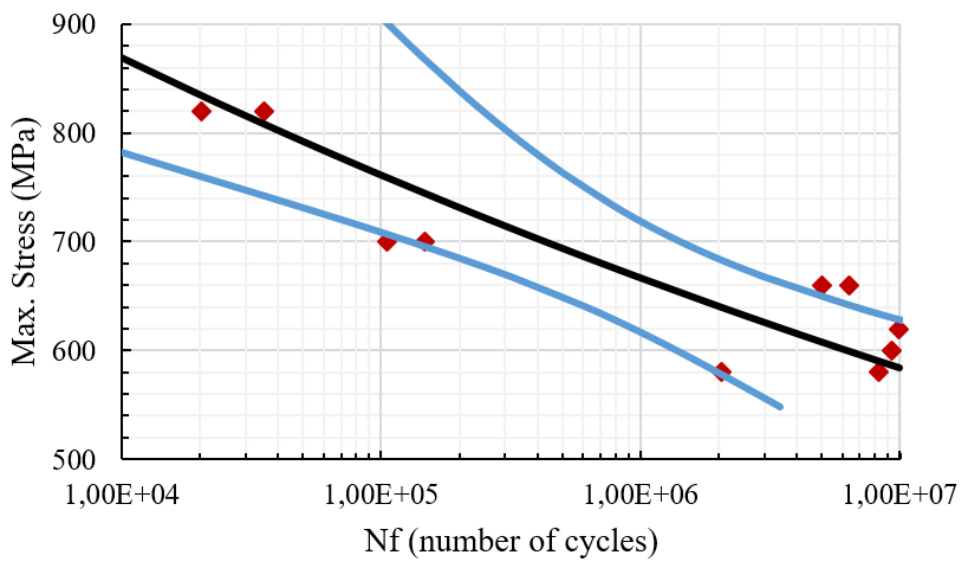

Figure 10: SN curve per ASTM E731-91 method

After calculation, mean value for $\% 50$ probability of failure in $10^{7}$ cycles (assumed as run-out criterion) was found 584.05 and $95 \%$ confidence bands were plotted in Fig. 10.

Kim and Zhang Method

Differently Kim and Zhang proposed fatigue damage concept while constructing SN curve. Accordingly, fatigue damage rate has a correlation with maximum stress and defined fitting parameters. By applying best fit between damage rate and stress in logarithmic base fitting parameters can be calculated. Only finite data points are used to estimate SN curve. Damage rate $\left(\Delta \mathrm{D}_{\mathrm{fi}} / \Delta \mathrm{N}_{\mathrm{fi}}\right)$ is calculated by given equation below:

$$
\begin{aligned}
& D_{f}=\left(1-\frac{S_{\text {max }}}{S_{\text {ultimate }}}\right) \\
& \frac{\Delta D_{f(i)}}{\Delta N_{f(i)}} \approx \alpha \cdot\left(S_{\max (i)}\right)^{\beta}
\end{aligned}
$$

where (i) represents each stress level on finite region and average life value can be taken in case of multiple test points on each stress level to present best fit relation. $\Delta D_{f(i)}=D_{f(i-1)}-D_{f(i)}$ for the case of $D_{f(i-1)}>D_{f(i)}$ and similarly $\Delta N_{f(i)}=N_{f(i-1)}-N_{f(i)} f$ for $N_{f(i-2}$ 1) $>N_{f(i)}$. 
To estimate fitting parameters $(\alpha$ and $\beta), \log \left(\Delta D_{f(i)} / \Delta N_{f(i)}\right)$ and $\log \left(S_{\max (i)}\right)$ are plotted and the slope gives $\beta$ value. Ten to power of intercept gives $\alpha$ value. After the parameters are calculated, $\mathrm{SN}$ curve is constructed with following equation:

$$
S_{\text {max }}=S_{u l t .} \cdot\left(\frac{\alpha \cdot(\beta-1) \cdot\left(N_{f}+N_{0}\right)}{S_{u l t .}^{-\beta}}+1\right)^{\frac{1}{1-\beta}}
$$

where $\mathrm{N}_{0}$ is the hypothetical value and indicates life of material under alternatingly loading. The corresponding $\mathrm{S}_{\max }$ for $\mathrm{N}_{0}$ equals to $S_{\text {ult }}$ and $\mathrm{N}_{0}$ is considered to be between 0 and 1 . After curve fitting, mean value for $\% 50$ probability of failure in 1.0E+07 cycles is $592.1 \mathrm{MPa}$ as shown in Fig. 11.

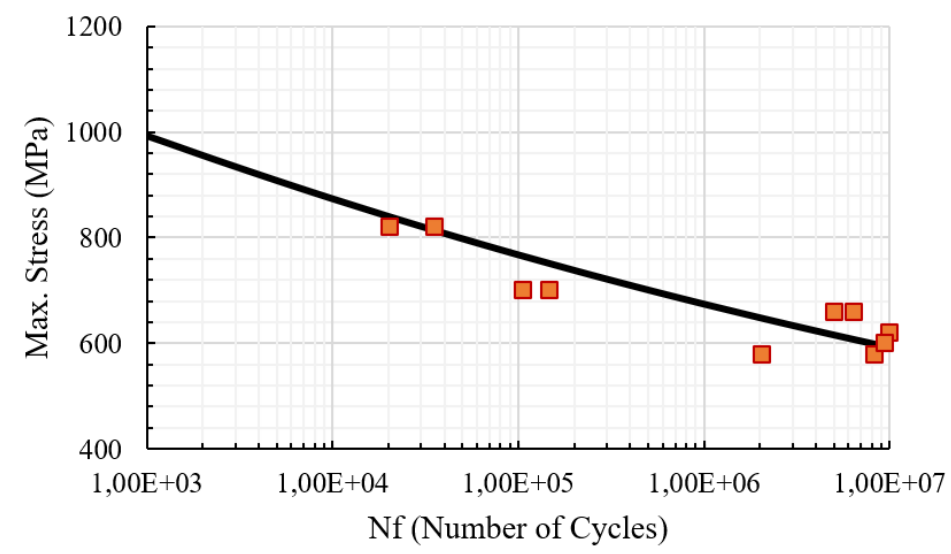

Figure 11: SN curve per Kim and Zhang method.

Bilinear Method

As an alternative method to random fatigue limit approach which optimizes parameters to get best-fit based on maximum likelihood method, bilinear random fatigue limit model is proposed [5]. It assumes that the SN curve consists of two distinct regions and linear region can be estimated by Basquin method via incorporating number of cycles in transition region and fatigue limit terms into stress equation:

$$
\begin{aligned}
& S=-m \cdot\left(\log N^{*}-N\right)+S_{\text {limit }} \text {. for } N<N^{*} . \\
& S=S_{\text {limit }} . \text { for } N \geq N^{*}
\end{aligned}
$$

where $m$ is the slope of curve in low cycle region and $N^{*}$ are the transition cycles from finite part to infinite part of SN curve.

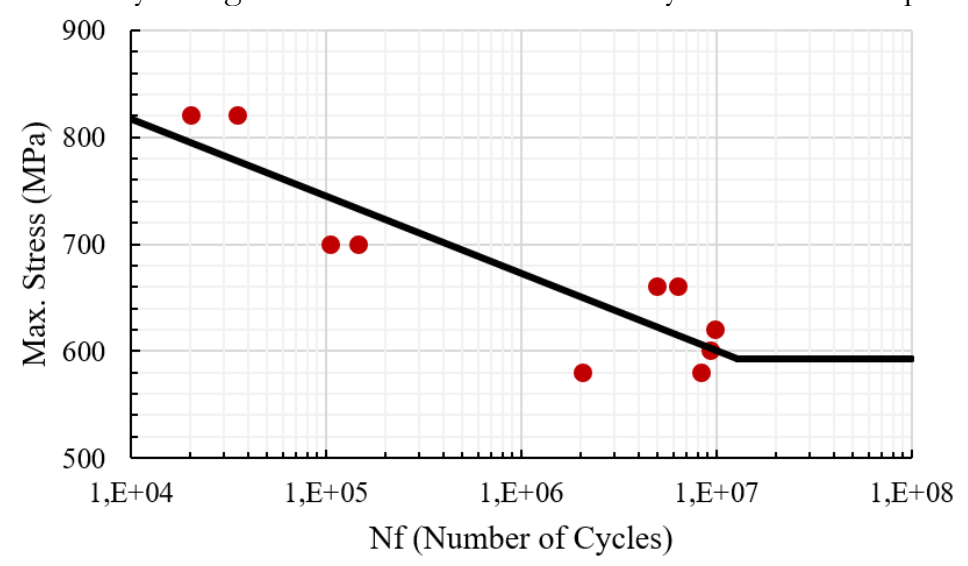

Figure 12: SN curve by Bilinear method 
Basically, it is recommended to use large experimental data for good fit. Therefore, it is a rough estimate of fatigue limit with small datasets because of not introducing smooth transition. Therefore, there is a weak correlation between high cycle and low cycles fatigue regions. Based on bilinear method, fatigue limit value for $50 \%$ probability of failure is $592 \mathrm{MPa}$ as shown in Fig. 12. Therefore, regression models provide less bias with strong estimation of fatigue data.

$\underline{\text { Stussi Method }}$

By addition of run-out data on data, non-linear models present unbiased estimation by linear regression. Stussi proposed a non-linear model to estimate SN curve in low and high cycle fatigue region by introducing tensile strength and fatigue stress at infinity into equation. This formulation is based on log-log scale and optimized by fitting parameters. Accordingly,

$$
S=\frac{R_{m}+\alpha \cdot N^{b} \cdot S_{\infty}}{1+\alpha \cdot N^{b}}
$$

where $R_{m}$ is the tensile strength of material, $a$ and $b$ are fitting parameters and $S_{\infty}$ is the infinite stress.

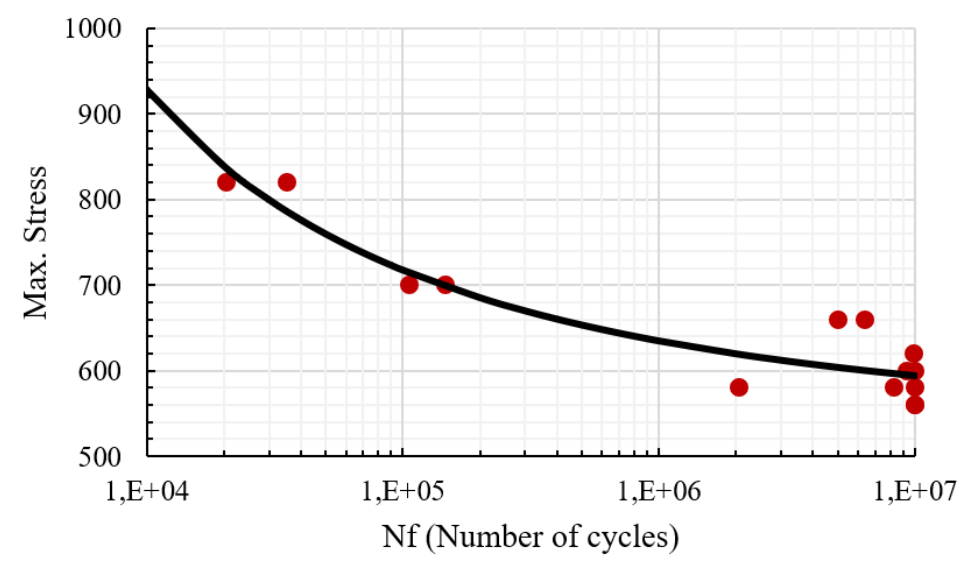

Figure 13: SN curve by Stussi estimation

This model is compatible with 3-parameter Weibull distribution and provides well-fitting not only on endurance region but also on low cycle fatigue region compared to previous methods specified. Based on this approach, mean value for $\% 50$ probability of failure which is decided as run-out criterion in $1.0 \mathrm{E}+07$ cycles is $594.6 \mathrm{MPa}$ as shown in Fig. 13.

AGARD-AG-292 Method

Another method is AGARD-AG-292, a comprehensive handbook for helicopter design, and sub-chapter 4.4 describes statistical analysis of small samples by presenting 4 parameters regression including censored data by considering constant scatter of fatigue data along all the regions of the SN curve. Accordingly, it is assumed that fatigue data is well fitted by Weibull distribution with four parameters fitting. Best fit is done with least square technique and log scale is used to make variable in linear form; however, increasing number of fitting parameters increases difficulties in calculating parameters. Extending curve beyond the data points cannot be conservative for endurance limit of material therefore data shall be used with reduced curve by introducing confidence interval.

$$
S=S_{\infty}+A \cdot(N+B)^{C}
$$

where $S$ is the stress, $S_{\infty}$ is infinite stress, $A, B$ and $C$ are constant fitting parameters, $N$ is the fatigue life of specimen at corresponding stress level.

Based on this approach, two-sided confidence interval (t-value of student variable) is used when estimating reduced curve. Accordingly, the mean and standard deviation are calculated assuming normal distribution of population. Then the variable $t_{\alpha}$ is calculated as given equation:

$$
\mu=\bar{x} \pm t_{\alpha} * \frac{s}{\sqrt{n-1}}
$$


To estimate $95 \%$ confidence interval for the population, SN curves are plotted for each specimen using same fitting parameters and ensuring passing over the observed test data for each specimen. Eventually, standard deviation is calculated on $10^{7}$ cycles (chosen as run-out criterion) with known stress points coming from each curve.

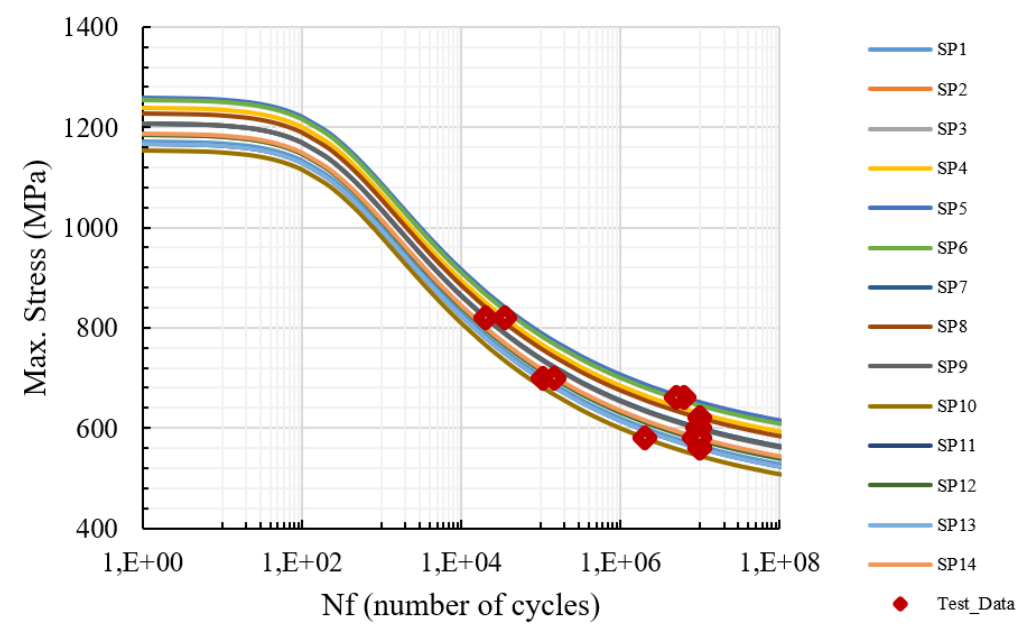

Figure 14: Constructing SN curve by AGARD-AG-292 method.

Accordingly, mean value with $50 \%$ probability of failure is $593.4 \mathrm{MPa}$ and standard deviation is 33.3 . Fatigue limit was estimated as $574.3 \mathrm{MPa}$ by applying $t_{\alpha}$-variable for $95 \%$ confidence interval.

\section{Fatigue Reliability Analysis}

It is important to present accurate data without any bias providing theoretical data after analysis that meets the requirement of desired lifetime of a component. Reliability is basically to intends to the probability of data without failure in real life conditions. Fatigue life evaluation through statical analysis is carried out to prevent fatigue failure under operation with accurate estimation. Negative relationship exists between fatigue life and reliability such that decreasing reliability results in increased fatigue life meaning that probability decreases and fatigue failure change increases.

SN curve can be generalized such that there is a linear relationship between life and applied stress in logarithmic base. By applying linear regression, model minimizes error by optimizing prediction. After estimation of observation, outliers shall be defined for reliable data by calculating residuals which is the difference between test data and prediction. For the sake of simplicity, standardized residuals are identified for each data point as corresponding error $\left(e_{i}\right)$ divided by an estimate of standard deviation.

$$
\begin{aligned}
& r_{i}=\frac{e_{i}}{\sqrt{\operatorname{MSE}\left(1-h_{i i}\right)}} \\
& M S E=\frac{1}{n} \sum_{i=1}^{n} e_{i}^{2} \\
& h_{i i}=\frac{1}{n}+\frac{\left(x_{i}-\bar{x}\right)^{2}}{\sum_{i=1}^{n}\left(x_{i}-\bar{x}\right)^{2}}
\end{aligned}
$$

where $r_{i}$ is the standardized residuals, $e_{i}$ is the standard error, $b_{i i}$ is leverage, $x_{i}$ is fatigue life of corresponding specimen, $\bar{x}$ is mean life of specimens and MSE is the mean square error. Outliers can be detected by looking at residuals and influential point is accepted an outlier if standardized residuals are greater than three in absolute value. Presence of potential outlier in data set influences regression model and results in biased predictor coefficients; therefore, studentized residuals are introduced by removing $i$-th observation from regression model. Then the formula becomes: 


$$
\begin{aligned}
& t_{i}=\frac{e_{i}}{\sqrt{M S E_{(i)}\left(1-h_{i i}\right)}} \\
& M S E_{i}=\left(M S E-\frac{e_{i}^{2}}{\left(1-h_{i i}\right)(n-k-1)}\right)\left(\frac{(n-k-1)}{(n-k-2)}\right)
\end{aligned}
$$

where $k$ is the number of independent variables, $n$ is the number of specimen and $e_{i}$ is the error between estimated and observed data. As shown in Fig. 15, there are no outliers for this data set.

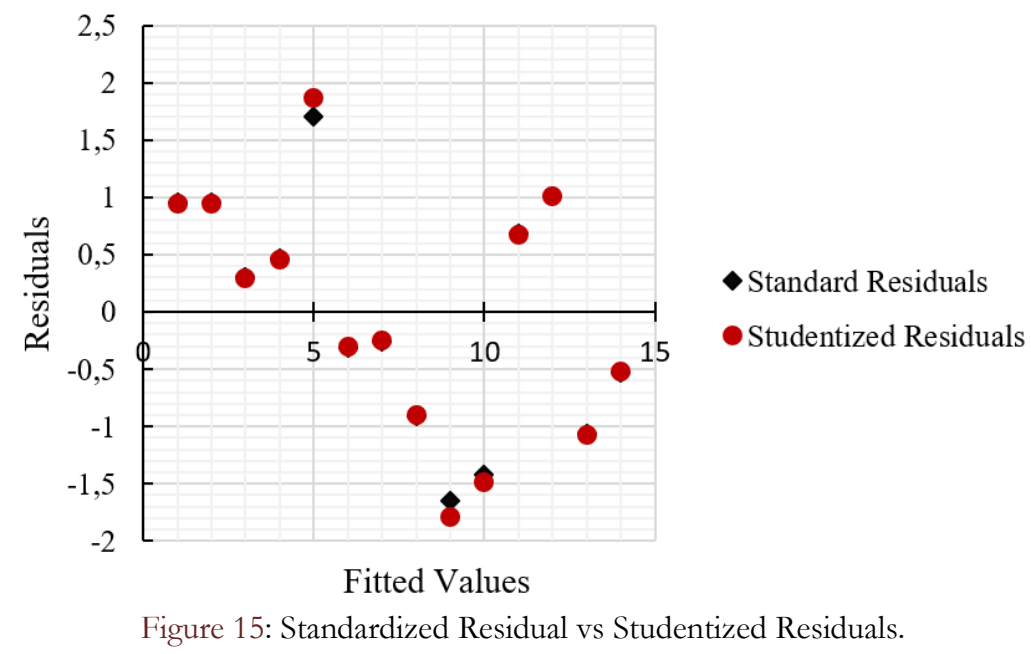

Another method to identify outliers is to construct normal probability plot. The points in line with normal probability plot line projects standard variation however outside of the line suspicious or outlier data. Normal probability plot is basically presenting relative cumulative rate of fatigue data. Samples are ordered in ascending manner and cumulative distribution function is calculated in the form of $F(S)=[(S-\mu) / \sigma]$ where $s, \mu$ and $\sigma$ are stress in logarithmic base, mean value and standard deviation respectively. Probability plot is constructed log-stress versus $z_{i}=F^{-1}\left(p_{i}\right)$ that is inverse function of cumulative distribution function. $\mathrm{P}_{\mathrm{i}}$ is called as plotting position and most used explanation proposed by Hazen [30] as follows:

$$
p_{i}=\frac{i-0.5}{n}
$$

Accordingly, theoretical data is plotted with 95\% confidence interval as shown in Fig. 16 and no outliers are found for normal probability plot.

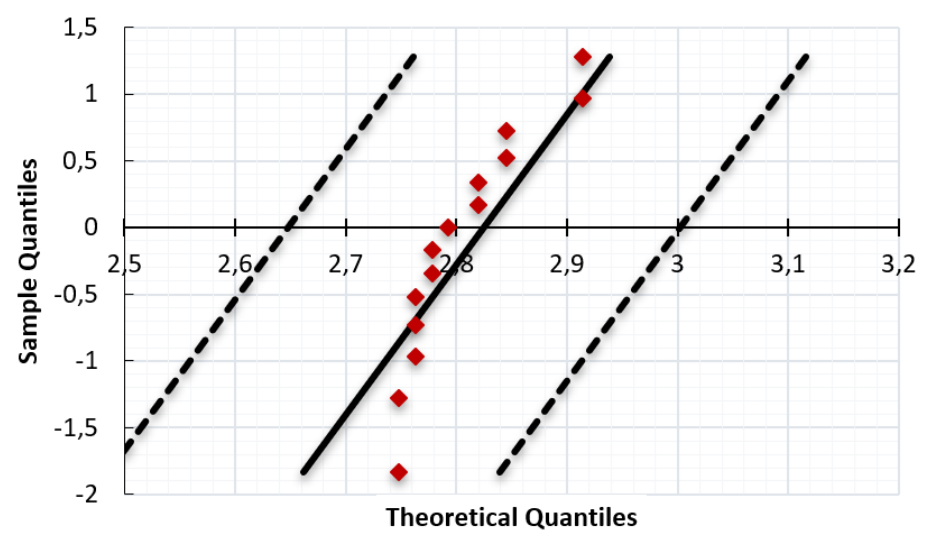

Figure 16: Normal Probability Plot. 
Fatigue data contains scatter because of nature of material and scatter band is covered by confidence interval. In the same manner, fatigue lifetime is identified at given probability of survival that determines necessary service time by assuming constant standard deviation though whole curve. Accordingly, fatigue curves for a probability of survival can be identified by destinating standard deviation following the probability of survival based on Gaussian log-normal distribution on given Tab. 2.

\begin{tabular}{cc}
\hline Probability of Survival $(\%)$ & Standard Deviation Factor \\
99.9 & -3 \\
97.7 & -2 \\
84 & -1 \\
50 & 0 \\
16 & 1 \\
2.3 & 2 \\
0.1 & 3 \\
\hline
\end{tabular}

Table 2: Probability of survival based on standard deviation.

It is explicit that having higher probability of survival eventuated higher reliability of data. In engineering applications SN curves are typically affiliated with $97,7 \%$ probability of survival meaning that offsetting from mean curve (50\%) by 2 times standard deviation to obtain fatigue data in a conservative way instead of 95\% probability of survival stated in ASTM E739$91[29]$.

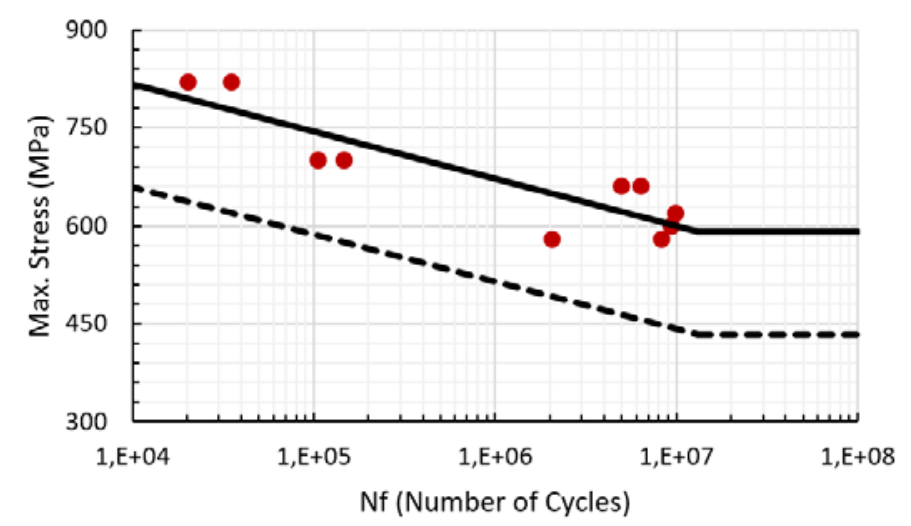

Figure 17: SN curve with probability of failure by Bilinear method

97.7\% probability of failure (survival) is generally used when estimating limits for design curves associated one-sided distribution. Mean and standard deviation of sample were considered same for entire population. Accordingly, design curve was constructed based on Bi-linear method as an example and $26.5 \%$ reduction in fatigue limit was observed as shown in Fig. 17.

$$
N_{97.7}=N_{50} \cdot 10^{-2 \cdot s}
$$

where $\mathrm{s}$ is the standard deviation and $\mathrm{N}_{50}$ is the life of the working curve over specified stress range.

\section{DISCUSSION}

$\mathrm{F}$ atigue damage in metals typically starts on surface and propagated perpendicular to applied load because of cyclic plasticity and isotropic properties of material. Fatigue mechanism behind of crack initiation is surface phenomenon on the other hand propagation is affiliated bulk phenomenon. Fatigue limit confirmed by SN curve acts as a threshold and below the endurance it allows crack initiation therefore it is affiliated with crack initiation phenomenon. Essentially it is not realistic to determine fatigue limit of material because of having randomly distribution of fatigue data however distribution of fatigue limit with correct bias can be estimated. To obtain good estimate of mean value with very 
small interval is only possible with handling large specimens while determining fatigue limit of material; otherwise, estimate will be biased in case of using small samples. Nevertheless, available methodologies proposed to advance accuracy of results with reduced number of specimens. Basically, staircase methods are easy to handle while estimating mean value of data set with $50 \%$ probability of failure; however, standard deviation is complicated because of small data set and needs to be severely reduced with safety factor to get conservative value. To analyze staircase test results, different methodologies was used by finding parameters of distribution that maximizes probability of test data. Maximum Likelihood method is the most efficient method in terms of estimation with less standard deviation compared to other methodologies. MLE usage gives advantage by providing closer confidence level to nominal ones compared to normal approximated intervals for staircase methods. It is advised to use stress increment is around $0.5 \sigma$ and $1.5 \sigma$ while performing staircase since small interval leads to waste observations on the other hand precision of mean decreases while increasing stress interval. Bootstrapping method resulted in too small standard deviation around mean that means less frequent occurrence and not presenting optimum confidence intervals. IABG method is better estimation than Dixon-Mood because of omitting first specimen' test results during analysis that means reduced sample size is used with bias correction. Also, Weibull distribution resulted in more suitable estimation for standard deviation compared to Dixon-Mood method. However, Dixon-Mood method gives best results in case of existence for very large coefficient of variance of data set. ASTM E739 is advised for metallic materials with the efficiency in medium and high cycle region, Stussi is effective also in low cycle fatigue region.

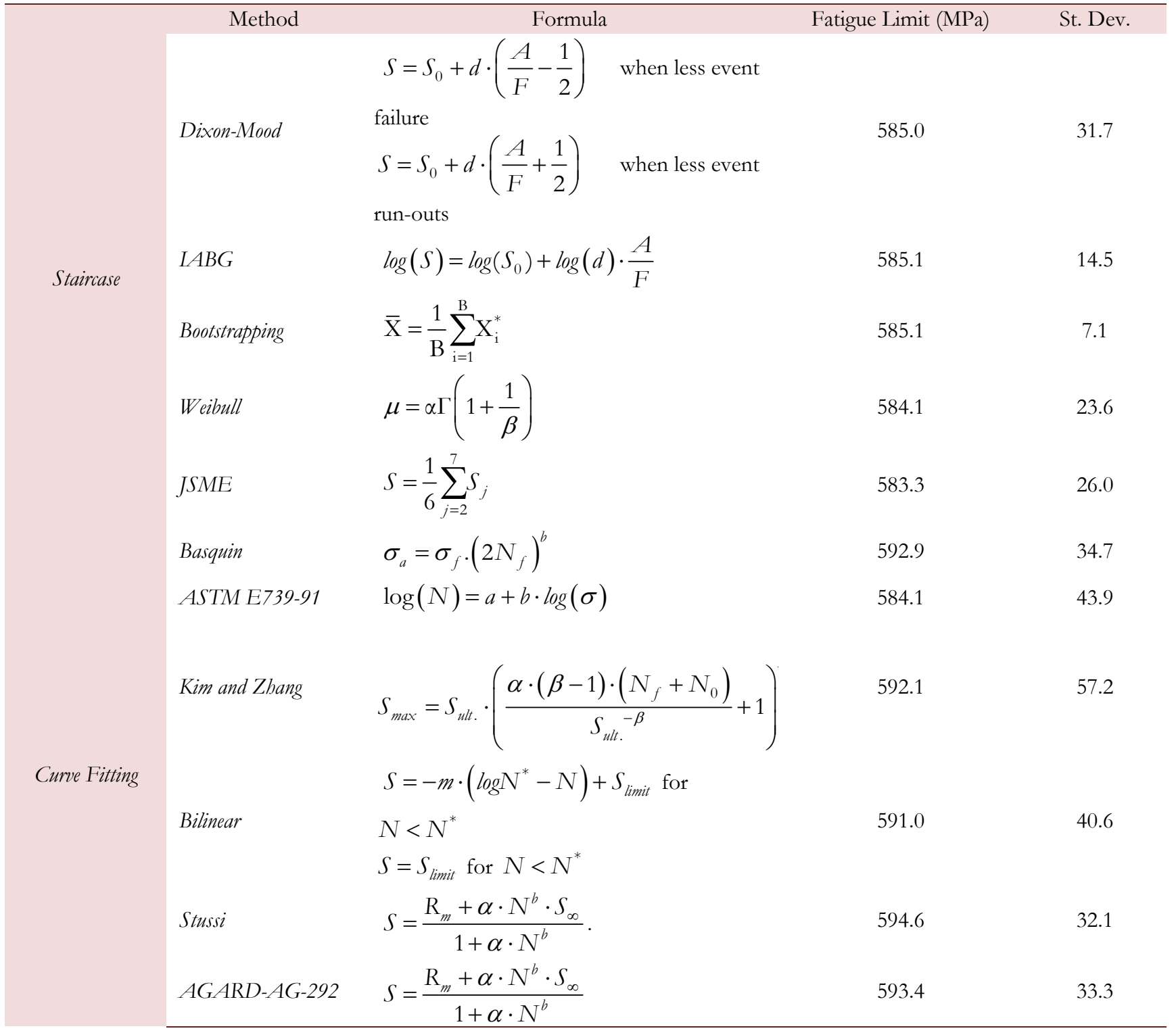

Table 3: Different approaches to determine fatigue limit of material. 
In engineering applications, it is accepted that fatigue strength and corresponding life follow log-normal or Weibull distribution; therefore, linear regression was operated to determine SN curve that allows estimation of fatigue limit as well. Curve fitting techniques were used in correlation between fatigue life and stress by optimizing fitting parameters. By increasing number of parameters to improve predictability of approach, solving equation becomes difficult because of increased iteration by least square technique and needs to be given necessary efforts. Linear regression gives mean value with $50 \%$ probability of failure and data needs to be reduced with defined probability of survival and confidence interval for resulting design data. If the presented distribution not fit data as desired, reliability estimation will be inexact. Basquin equation estimated poorly because of limited capabilities in case of addition of ultimate strength of data as an input that regression models provide good estimation with goodness of fit. Bilinear model can be used for materials showing fatigue behavior of sharp transition between infinite and finite region with relatively constant scatter though SN curve. Other models exhibited closer estimation for medium and high cycle fatigue region. Exceptionally Kim and Zhang resulted in high covariance because of limited data on low cycle fatigue region such that this method is more suitable for stress level higher than four on finite region. Nevertheless, prediction of fatigue limit data agreed with observed data sensibly.

Scatter band is caused by inhomogeneous defect size in microstructure of material, production, environment, operator, and misalignment of test specimen on machine [6]. In general scatter is observed larger on low cycle fatigue region and lower on infinite region of SN curve. Probability distribution of fatigue data was studied for reliability assessment. Inverse correlation exists between fatigue life and reliability, fatigue reliability decreases when increased fatigue life. After estimation of fatigue data in terms of stress and life relationships, residual analysis needs to be performed for reliable data in structural design. In this point, outliers can be detected to present more accurate data by employing standard deviation for normal probability plot and standard/ studentized residuals which is calculated by least square estimation based on minimizing sum of squared deviations. Significance level denoted as 0.05 meaning that $5 \%$ risk of data and confidence level and probability of survival for fatigue lifetime was calculated for $95 \%$ and 97.7 respectively for this study.

In this paper, it was attempted to determine endurance limit of material using staircase and curve fitting methods and their results were tabulated on Tab. 3.

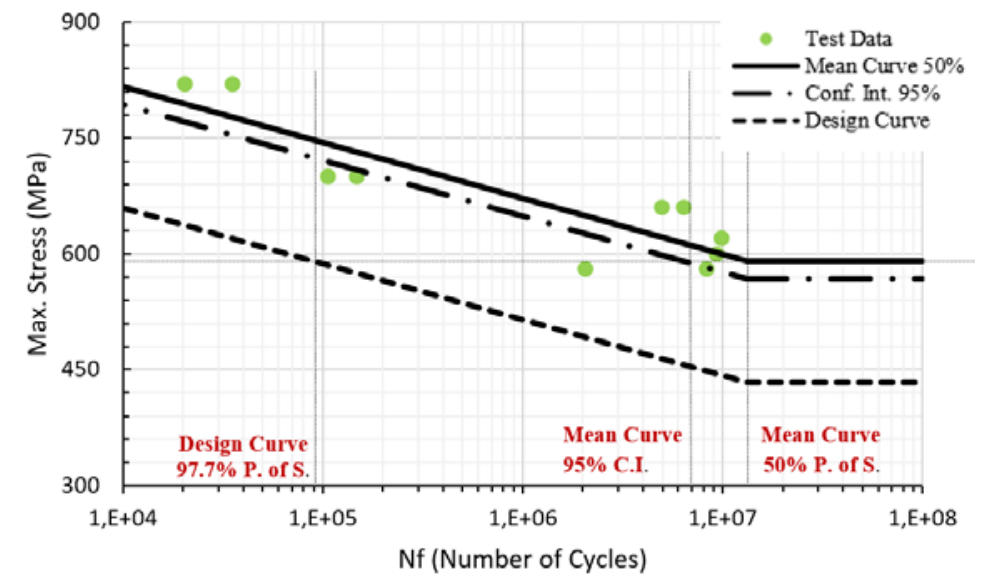

Figure 18: Variation of design curve after probabilistic analysis (C.I.: Confidence Interval, P. of S.: Probability of Survival).

To qualify fatigue test data coming from coupon tests, design curves need to be constructed by applying reduction factor to present conservative data as shown in Fig. 18. In this way, probabilistic nature of fatigue data can accurately be estimated accounting for scatter effects with confidence.

\section{CONCLUSIONS}

$\mathrm{F}$ atigue strength of a material can be determined by evaluation of staircase or curve fitting methods statistically. The idea is to present more accurate data with specified confidence intervals such that conservative data needs to be used in design side. The following conclusions drawn from this study are listed below:

- Weibull and $I A B G$ method resulted in better fit compared to other methods in terms of fatigue limit and standard deviation for limited data set. 
- Total number of specimens (14) seems appropriate to construct SN curve, 8 of which are finite and 6 for infinite regions which makes it possible to determine endurance limit for small sample data analysis.

- Even though analyses have been carried out for AISI 4340 coupon test data, it can be commissioned for any material that exhibits fatigue limit behavior.

- Staircase method is easy to operate for estimating fatigue limit of material and proposed methods exhibit similar results in terms of $50 \%$ probability of failure; however, standard deviation is complicated so large safety factors are inevitable to get reliable results for small samples.

- Scatter band of SN curve at 95\% confidence level and $97.7 \%$ probability of survival needs to be studied for accurate and reliable data for design curves.

- Curve fitting methods can be used to determine SN curve shape by allowing estimation of fatigue strength for corresponding number of cycles and estimated fatigue limit at $1 \mathrm{E}+07$ cycles resulted in close agreement with staircase methods.

- Coupon level testing is carried out in laboratory conditions and results give an idea about fatigue behavior of material; however, components in service can have unforeseen risks and this is not easy to estimate in terms of scatter therefore additional safety (knock-down) factors are needed.

\section{ACKNOWLEDGMENTS}

A

uthors acknowledge Turkish Aerospace Inc.

\section{REFERENCES}

[1] Burhan, I., and Kim, H. (2018). S-N curve models for composite materials characterisation: An evaluative review. Journal of Composites Science, 2(3), 38. DOI: 10.3390/jcs2030038.

[2] Murakami, Y., Takagi, T., Wada, K., and Matsunaga, H. (2021). Essential structure of S-N curve: Prediction of fatigue life and fatigue limit of defective materials and nature of scatter. International Journal of Fatigue, 146, 106138. DOI: 10.1016/j.ijfatigue.2020.106138.

[3] Barbosa, J. F., Correia, J. A. F. O., Freire Júnior, R. C. S., Zhu, S.-P., and De Jesus, A. M. P. (2019). Probabilistic s-n fields based on statistical distributions applied to metallic and composite materials: State of the art. Advances in Mechanical Engineering, 11(8), 168781401987039. DOI: 10.1177/1687814019870395.

[4] Leonetti, D., Maljaars, J., and Snijder, H. H. (B. (2017). Fitting fatigue test data with a novel s-n curve using frequentist and Bayesian inference. International Journal of Fatigue, 105, pp. 128-143. DOI: 10.1016/j.ijfatigue.2017.08.024.

[5] Pollak, R., Palazotto, A., and Nicholas, T. (2006). A simulation-based investigation of the staircase method for fatigue strength testing. Mechanics of Materials, 38(12), pp. 1170-1181. DOI: 10.1016/j.mechmat.2005.12.005.

[6] Schijve, J. (2005). Statistical distribution functions and fatigue of structures. International Journal of Fatigue, 27(9), pp. 1031-1039. DOI: 10.1016/j.ijfatigue.2005.03.001.

[7] Pascual, F. G., and Meeker, W. Q. (1999). Estimating fatigue curves with the random fatigue-limit model. Technometrics, 41(4), pp. 277-289. DOI: 10.1080/00401706.1999.10485925.

[8] Rabb, B.R. (2003). Staircase testing - confidence and reliability. Trans. Eng. Sci. 40, 447-464.

[9] Sutherland, H. and Veers, P. (2000). The Development of confidence limits for fatigue strength data. Wind Energy ASME/AIAA. 10.2514/6.2000-63.

[10] Sakai, T., Nakajima, M., Tokaji, K., and Hasegawa, N. (1997). Statistical distribution patterns in mechanical and fatigue properties of metallic materials. Journal of the Society of Materials Science, Japan, 46(6Appendix), pp. 63-74. DOI: 10.2472/jsms.46.6appendix_63.

[11] Li, H., Wen, D., Lu, Z., Wang, Y., and Deng, F. (2016). Identifying the probability distribution of fatigue life using the maximum entropy principle. Entropy, 18(4), 111. DOI: 10.3390/e18040111.

[12] Schneider, C. R. A., and Maddox, S. J. (2003). Best practice guide on statistical analysis of fatigue data. The Welding Institute, Cambridge, UK. 
[13] Toasa C., and Ummenhofer, T. (2018). A probabilistic Stüssi function for modelling the S-N curves and its application on specimens made of steel S355J2+N. International Journal of Fatigue. 117. DOI: 10.1016/j.ijfatigue.2018.07.041.

[14] Kim, H. S., and Zhang, J. (2001). Fatigue damage and life prediction of glass/vinyl ester composites. Journal of Reinforced Plastics and Composites, 20(10), pp. 834-848. DOI: 10.1177/073168401772678959.

[15] Lipski, A. (2018). Determination of the S-N curve and the fatigue limit by means of the thermographic method for ductile cast iron. DOI: $10.1063 / 1.5066398$.

[16] Dixon, W. J., and Mood, A. M. (1948). A method for obtaining and analyzing sensitivity data. Journal of the American Statistical Association, 43(241), pp. 109-126. DOI: 10.1080/01621459.1948.10483254.

[17] Dixon, W. J. (1965). The up-and-down method for small samples. Journal of the American Statistical Association, 60(312), pp. 967-978. DOI: 10.1080/01621459.1965.10480843.

[18] Lin, S. (2001). Evaluation of the staircase and the accelerated test methods for fatigue limit distributions. International Journal of Fatigue, 23(1), pp. 75-83. DOI: 10.1016/s0142-1123(00)00039-6.

[19] Little, R. E. (1972). Estimating the median fatigue limit for very small up-and-down quantal response tests and for. Probabilistic Aspects of Fatigue. DOI: 10.1520/stp35403s.

[20] Loren, S. (2003). Fatigue limit estimated using finite lives. Fatigue Fracture of Engineering Materials and Structures, 26(9), pp. 757-766. DOI: 10.1046/j.1460-2695.2003.00659.x.

[21] Minak, G. (2007). Comparison of different methods for fatigue limit evaluation by means of the monte carlo method. Journal of Testing and Evaluation, 35(2), 100122. DOI: 10.1520/jte100122.

[22] Nicholas M., and Ulam S. (1949). The monte carlo method, Journal of the American Statistical Association, 44, pp. 335341

[23] Johnson, R. W. (2001). An introduction to the bootstrap. Teach. Stat. 23, pp. 49-54.

[24] Beretta, S., and Murakami, Y. (1998). Statistical analysis of defects for fatigue strength prediction and quality control of materials. Fatigue Fracture of Engineering Materials and Structures, 21(9), pp. 1049-1065.

DOI: 10.1046/j.1460-2695.1998.00104.x.

[25] Wallin, K. R. W. (2011). Statistical uncertainty in the fatigue threshold staircase test method. International Journal of Fatigue, 33(3), pp. 354-362. DOI: 10.1016/j.ijfatigue.2010.09.013.

[26] Hück, M. (1983). Ein verbessertes verfahren für die auswertung von treppenstufenversuchen. Materialwissenschaft Und Werkstofftechnik, 14(12), 406-417. DOI: 10.1002/mawe.19830141207.

[27] Müller, C., Wächter, M., Masendorf, R., and Esderts, A. (2017). Accuracy of fatigue limits estimated by the staircase method using different evaluation techniques. International Journal of Fatigue, 100, pp. 296-307. DOI: $10.1016 /$ j.ijfatigue.2017.03.030.

[28] Efron, B., and Tibshirani, R. (1986). Bootstrap methods for standard errors, confidence intervals, and other measures of statistical accuracy: Rejoinder. Statistical Science, 1(1). DOI: 10.1214/ss/1177013815.

[29] ASTM E739-91 (1998). Available at: https://www.astm.org/DATABASE.CART/HISTORICAL/E739-91R98.htm.

[30] Hazen, A. (1914). Storage to be provided in impounding municipal water supply. Transactions of the American Society of Civil Engineers, 77(1), pp. 1539-1640. DOI: 10.1061/taceat.0002563.

[31] Beretta, S., Clerici, P., and Matteazzi, S. (1995). The effect of sample size on the confidence of endurance fatigue tests. Fatigue and Fracture of Engineering Materials and Structures, 18(1), pp. 129-139.

DOI: $10.1111 / j .1460-2695.1995 . t b 00147 . x$.

[32] Molina, A., Piña-Monarrez, M. R., and Barraza-Contreras, J. M. (2020). Weibull S-N fatigue Strength curve analysis for a572 Gr. 50 Steel, based on the True stress_-true Strain Approach. Applied Sciences, 10(16), 5725. DOI: $10.3390 /$ app10165725.

[33] Engler-Pinto, C. C., Lasecki, J. V., Frisch, R. J., Dejack, M. A., and Allison, J. E. (2005). Statistical approaches applied to fatigue test data analysis. SAE Technical Paper Series. DOI: 10.4271/2005-01-0802.

[34] Zheng, X., Lü, B., and Jiang, H. (1995). Determination of probability distribution of fatigue strength and expressions of P-S-N curves. Engineering Fracture Mechanics, 50(4), pp. 483-491. DOI: 10.1016/0013-7944(94)00218-7

[35] Boylan, G. L., and Cho, B. R. (2011). The normal probability plot as a tool for understanding data: a shape analysis from the perspective of skewness, kurtosis, and variability. Quality and Reliability Engineering International, 28(3), pp. 249264. DOI: 10.1002 /qre.1241.

[36] Sivák, P., and Ostertagová, E. (2012). Evaluation of fatigue tests by means of mathematical statistics. Procedia Engineering, 48, pp. 636-642. DOI: 10.1016/j.proeng.2012.09.564

[37] Jamalkhani K., M., and Azadi, M. (2018). Reliability prediction, scatter-band analysis and fatigue limit assessment of high-cycle fatigue properties in en-gjs700-2 ductile cast iron. MATEC Web of Conferences, 165, 10012.

DOI: $10.1051 /$ matecconf/201816510012 
[38] Najim, A., and Anwar T. (2015). Comparison four methods for estimating the fatigue life distribution parameters through simulation. International Journal of Science and Research, pp. 2319-7064.

[39] AGARD-AG-292 (1983). Helicopter fatigue design guide.

[40] Kepka, M. (2018). Using design SN curves and design stress spectra for probabilistic fatigue life assessment of vehicle components.

[41] Fan, J. L., Guo, X. L., Wu, C. W., and Zhao, Y. G. (2011). Research on fatigue behavior evaluation and fatigue fracture mechanisms of cruciform welded joints. Materials Science and Engineering: A, 528(29-30), pp. 8417-8427. DOI: $10.1016 /$ j.msea.2011.08.037.

[42] ASTM E466-96 (1996). Standard practice for conducting force controlled constant amplitude axial fatigue tests of metallic materials, ASTM International, West Conshohocken, PA, Available at: www.astm.org.

[43] AMS 2759-1D (2007). Heat treatment of carbon and low-alloy steel parts minimum tensile strength below $220 \mathrm{ksi}$ (1517 $\mathrm{MPa})$, SAE International. 